\title{
Algorithmic Study of the Characteristics of Electrocardiograph Signals in Patients with Coronary Heart Disease
}

\author{
Honger Li $(D)$ and Lixia Zhao \\ Electrocardiographic Room, Zhejiang Xiaoshan Hospital, Hangzhou 311200, China \\ Correspondence should be addressed to Lixia Zhao; 1410601313@stu.cque.edu.cn
}

Received 22 June 2021; Revised 5 August 2021; Accepted 6 August 2021; Published 6 September 2021

Academic Editor: Gustavo Ramirez

Copyright ( $\odot 2021$ Honger Li and Lixia Zhao. This is an open access article distributed under the Creative Commons Attribution License, which permits unrestricted use, distribution, and reproduction in any medium, provided the original work is properly cited.

\begin{abstract}
This work aimed to analyze the electrocardiogram (ECG) characteristics and signal classification of patients with coronary heart disease (CHD) diagnosed by coronary angiography, so as to provide a theoretical basis for the clinical adoption of ECG images. 106 patients with CHD who were admitted to the XXX hospital from January 15, 2019, to May 30, 2020, underwent coronary intervention therapy, and their ECG indicators were recorded during the operation. Then, the LetNet-SoM algorithm designed in this work, as well as the traditional algorithms GoogLeNet and SqueezeNet, was applied to the patient's ECG classification. It was found that part of ECG wave (QRS) and corrected Q-T interval (QTC) of patients after treatment were higher than those before treatment $(P<0.05)$, but PR interval, RR interval, Tpeak-Tend $(\mathrm{TpTe})$ interval, and QT interval were not substantially different from those before treatment $(P>0.05)$. The diagnostic accuracy, sensitivity, and specificity of LetNet-SoM algorithm for patients with CHD were better than those of traditional algorithms, with evident difference $(P<0.05)$. The classification time of LetNetSoM algorithm was lower in contrast to that of traditional algorithms, and the difference was also notable $(P<0.05)$. The $\mathrm{R}$ wave and $\mathrm{T}$ wave indicators of patients after treatment were higher than before treatment, with notable difference $(P<0.05)$. The difference between the patient's $S$ wave indicator before and after treatment was not statistically significant $(P>0.05)$. The positive rate of S wave amplitude, QRS, and QTC was $68.15 \%, 60.52 \%$, and $51.36 \%$, respectively. In short, the LetNet-SoM algorithm designed based on lightweight neural network had excellent performance in classification and diagnosis of ECG, and it had the value of further popularization and application. The ECG signals were important indicators in the diagnosis of CHD, among which the S wave amplitude, QRS, and QTC were the most sensitive ones.
\end{abstract}

\section{Introduction}

The blood supply to the heart is not static but always fluctuates, and this fluctuation needs to be adjusted by the body itself, to promote the relatively constant blood supply and ensure the normal work of the heart [1]. If any kind of cause causes myocardial ischemia, the body's regulation will not meet the needs of the heart, which constitutes the true myocardial ischemia [2]. Therefore, myocardial ischemia means that the blood perfusion of the heart is reduced, resulting in ischemia and hypoxia of the heart, which cannot meet the normal energy requirements of the heart. Mild myocardial ischemia can compensate for the heart, and the symptoms are not obvious. The symptoms of chest tightness, shortness of breath, palpitations, and fatigue may appear after physical labor, which will be relieved after rest. The more severe ones can cause angina, which is the compressive pain behind the breastbone. It can radiate to the left shoulder, left upper arm, and other parts. The patient will often experience symptoms such as difficulty breathing, sweating, nausea, and vomiting due to severe pain. It even causes myocardial infarction, threatening the lives of patients $[3,4]$. There are many reasons for myocardial ischemia, such as decreased blood volume, coronary artery obstruction, coronary heart disease, and valvular heart disease. Therefore, how to diagnose myocardial ischemia as early as possible is of great significance to the prognosis of patients.

Coronary angiography can show the location, scope, and degree of coronary artery disease. It has diagnostic value for the 
diagnosis of asymptomatic myocardial ischemia. Moreover, it is the gold standard recognized by the medical community, but it is minimally invasive for patients. Resting or exercise echocardiography can show localized abnormal wall motion caused by myocardial ischemia, but it is easily affected by the patient's ventilation and has low specificity. Holter is currently the best approach to study painless ischemia, and it has the advantages of being noninvasive, simple, accurate, real-time, repeatable, and quantifiable. Moreover, it can accurately reflect the frequency, duration, severity, and dynamic changes of myocardial ischemia [5]. Valensi and Meune [6] obtained echocardiograms of patients with type 2 diabetes for analysis, with the coronary artery results as the standard; it was found that cost-effectiveness of asymptomatic screening for myocardial ischemia as part of a risk assessment for heart failure can contribute to the preventive treatment of asymptomatic patients with myocardial ischemia. West et al. [7] used ECG gated chestcomputed tomography to scan patients with coronary heart disease and compared them with the results of coronary angiography. It was found that ECG features had high accuracy, sensitivity, and specificity in the diagnosis of coronary heart disease. As the level of deep learning neural networks becomes deeper and deeper, there are increasing parameters, and its requirements for hardware are getting higher and higher. Moreover, it has been unable to meet the requirements of light and compact embedded hardware such as mobile phones and computing platforms for autonomous driving. Therefore, many scholars focus on how to streamline and optimize the network model, so that they can run more smoothly on embedded devices with limited hardware conditions, and lightweight neural networks came into being. Therefore, it intends to introduce a new type of ECG signal classification algorithm designed by neural network convolution.

In summary, the ECG signal classification algorithm LetNet-SoM was constructed based on the lightweight neural network. The patient's coronary angiography results were taken as the gold standard, and it was compared with GoogLeNet and SqueezeNet algorithms. It was then applied in the ECG diagnosis of 106 patients with myocardial ischemia diagnosed as coronary heart disease by coronary angiography in the hospital. After comparing the ECG interval and amplitude indicator before and after treatment, the clinical diagnostic value of ECG in patients with myocardial ischemia was comprehensively evaluated based on ECG signal classification algorithm and coronary angiography.

\section{Materials and Methods}

2.1. Research Subjects. 106 patients admitted to the hospital from January 15, 2018, to May 30, 2020, who were diagnosed as coronary heart disease by coronary angiography were selected as study subjects, all of which received coronary intervention therapy. The ECG was recorded by the monitoring system of multilead ECG during operation. The study had been approved by the Medical Ethics Committee of xxx Hospital, and the patients and their families understood the meaning of the study and had signed an informed consent form.

Inclusion criteria were the following: (1) patients with symptoms of myocardial ischemia; (2) samples of patients who voluntarily received interventional therapy; (3) patients with similar symptoms such as angina caused by activities; (4) patients with triple vessel disease and Syntax score [8] less than 22 points; (5) patients with the coronary artery diameter greater than $70 \%$.

Exclusion criteria were the following: (1) patients with liver and kidney diseases; (2) patients who had reached the end stage of heart failure; (3) patients with electrolyte disturbance; (4) patients with dilated myocardial disease.

\subsection{ECG Signal Classification Algorithm Based on Lightweight} Neural Network. Traditionally, the ECG diagnosis obtained by traditional devices is mainly classifying new data by extracting abnormal features of the ECG. But, if the feature itself is not universal or specific, it is difficult to make a clear classification. Therefore, it first introduces the latest lightweight neural network to design algorithm. Then, it is put it into a restricted mobile device to realize feature extraction and classification of ECG data. In Figure 1, the obtained ECG data can be input to a mobile device, and the designed algorithm can be used to classify and output the corresponding label.

Based on the above ideas, the traditional two-dimensional convolution is changed to a one-dimensional convolution structure, and then the one-dimensional convolution equation can be expressed as follows:

$$
y[p]=x[p] * g[p]=\sum_{k=0}^{q-1} x[k] g[p-k] .
$$

In (1), $y[p]$ represents the output sequence, $x[p]$ represents the input sequence, $q$ represents the convolution length, and $g[p]$ represents the convolution kernel. It is supposed that the input sequence is $x[p]=\left[x_{1}, x_{2}, x_{3}\right]$ and the convolution kernel is $g[p]=\left[g_{1}, g_{2}, g_{3}\right]$; then the output sequence can be expressed as follows:

$$
\begin{aligned}
& y[0]=x[0] * g[0-0]+x[1] * g[0-1]+x[2] * g[0-2]=g_{1} * x_{1}, \\
& y[1]=x[0] * g[1-0]+x[1] * g[1-1]+x[2] * g[1-2]=g_{1} * x_{2}+g_{2} * x_{2}, \\
& y[2]=x[0] * g[2-0]+x[1] * g[2-1]+x[2] * g[2-2]=g_{1} * x_{3}+g_{2} * x_{2}+g_{3} * x_{1},
\end{aligned}
$$




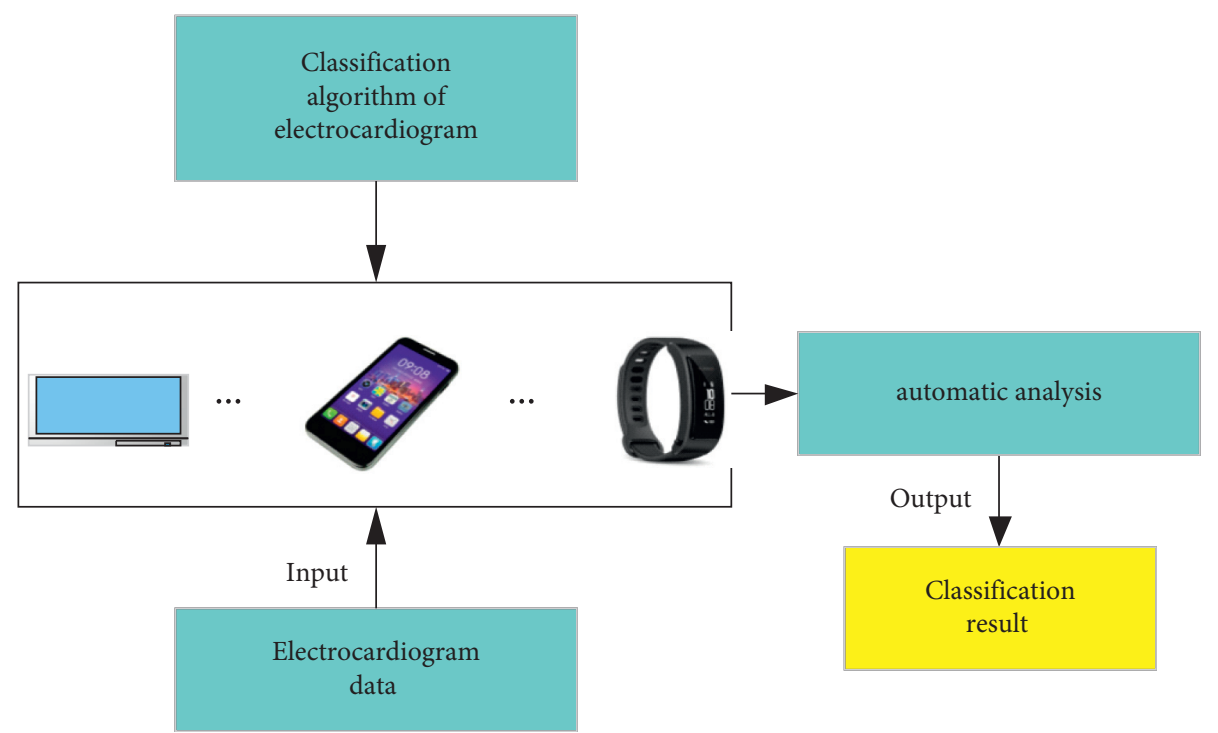

FIGURE 1: Schematic diagram of the overall concept of the constructed algorithm.

$$
\begin{aligned}
& y[3]=x[0] * g[3-0]+x[1] * g[3-1]+x[2] * g[3-2]=g_{2} * x_{3}+g_{3} * x_{2}, \\
& y[4]=x[0] * g[4-0]+x[1] * g[4-1]+x[2] * g[4-2]=g_{3} * x_{3} .
\end{aligned}
$$

In (2)-(6), the length of the output sequence is 5 , and $y[0], y[1], y[2], y[3]$, and $y[4]$ can be output sequentially. In the whole algorithm, the Lite module [9] is proposed as the processing core of the model in Figure 2. This module uses the $1 \times 1$ convolution method, which can pass the convolution of $1 \times 1,1 \times 2$, and $1 \times 3$ in parallel channel. A layer of volume is added to the convolution channel of $1 \times 2$ and $1 \times 3$, deep learning can be adopted to separate the convolution structure, and a residual connection is also designed.

For the multiclassification problem, it introduces the neural network Softmax as the classifier, the classification target is set to 5 , and then the Softmax classifier requires 5 neurons, which can be expressed as follows:

$$
\sum_{i}^{5} h_{i}=1 \text {. }
$$

In (7), $i=1,2, \ldots, 5$, and $h_{i}$ represents the probability distribution of different neurons. Then, it is supposed that the weight of the previous layer connected to Softmax is $W$, and the input of the Softmax classifier can be expressed as follows:

$$
z_{i}=\sum_{k} x_{k} W_{k i}
$$

In (8), $z_{i}$ represents the input sequence of the Softmax classifier, and the probability that the Softmax classifier calculates and outputs 5 neurons is as follows:

$$
h_{i}=\frac{\exp \left(z_{i}\right)}{\sum_{j}^{5} \exp \left(z_{i}\right)} \text {. }
$$

The final predicted output category can be expressed as follows:

$$
\widehat{i}=\arg \max h_{i}=\arg \max z_{i} .
$$

In (10), $\hat{i}$ represents the final predicted output category. In order to express the similarity between the predicted category and the true category, it introduces cross entropy as the loss function, which can be expressed as follows:

$$
F(r, s)=-\sum_{x} r(x) \log s(x)
$$

In (11), $F(r, s)$ represents the cross entropy, $r$ represents the expected label output, $s$ represents the true label output, $r(x)$ represents the probability of the expected label output, and $s(x)$ represents the probability of the true label output. It is assumed the sequence is $p=3$; then the expected output is $r=\left(r_{1}, r_{2}, r_{3}\right)$, and the real outputs are $p=\left(p_{1}, p_{2}, p_{3}\right)$ and $q=\left(q_{1}, q_{2}, q_{3}\right)$, so the following equations can be obtained:

$$
F(r, p)=-\left(r_{1} * \log ^{p_{1}}+r_{2} * \log ^{p_{2}}+r_{3} * \log ^{p_{3}}\right)=k_{1},
$$

$$
F(r, q)=-\left(r_{1} * \log ^{q_{1}}+r_{2} * \log ^{q_{2}}+r_{3} * \log ^{q_{3}}\right)=k_{2} .
$$

In (12) and (13), if $k_{1}<k_{2}, k_{1}$ is close to the expected output; otherwise $k_{2}$ is close to the expected output. The smaller the loss function value, the better the classification effect. The above is an ECG signal classification algorithm based on a lightweight neural network (set as LetNetSoM). 


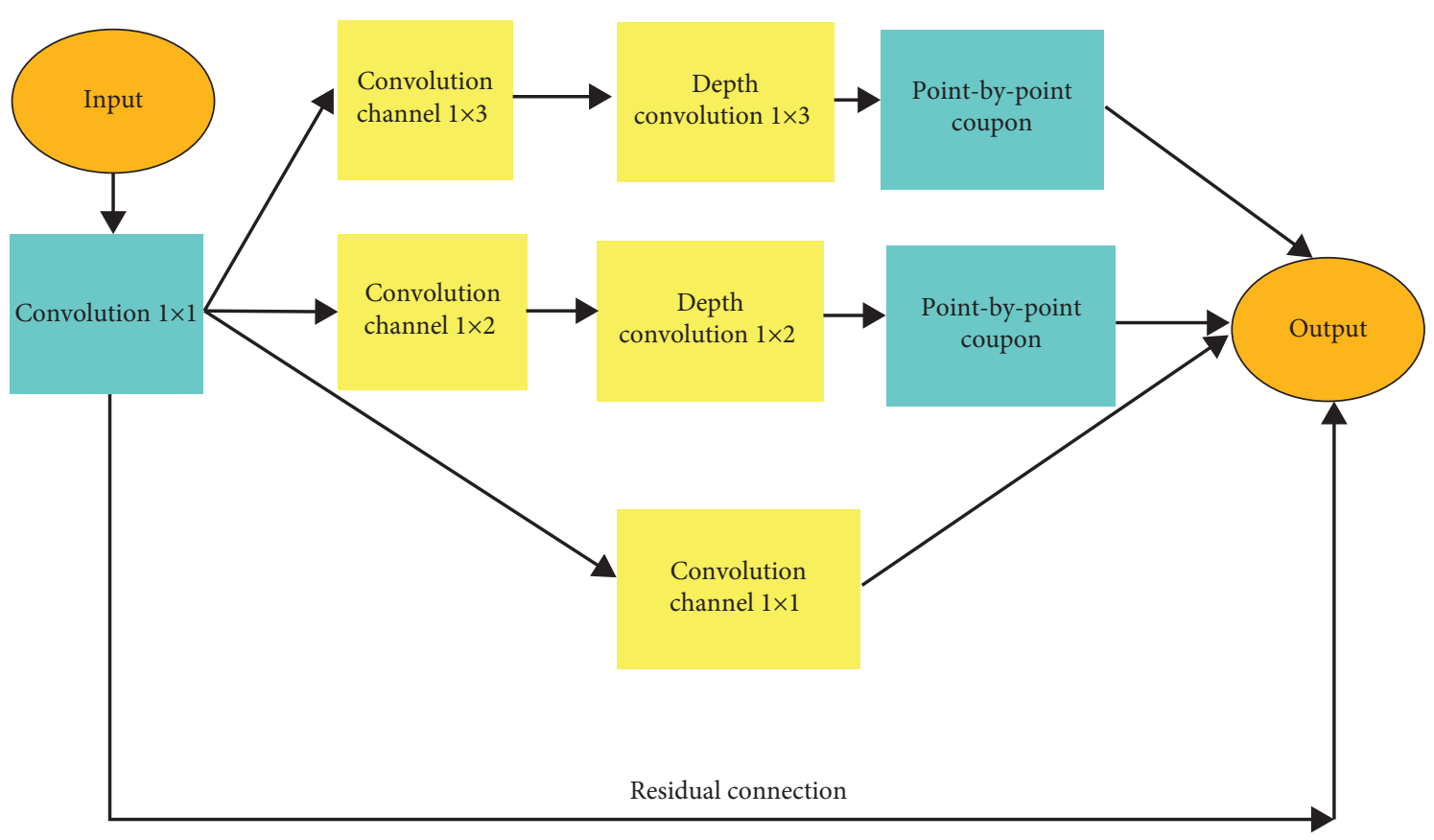

FIGURE 2: Schematic diagram of the Lite module structure.

2.3. ECG Data Collection. According to the results of coronary angiography, appropriate interventional instruments were selected. Before treatment, the disposable light-permeable electrode pads were placed on the standard ECG lead electrodes on the patient's body surface. After the electrophysiological instrument of Bard was switched on, the ECG of the patient was continuously collected until the end of the operation. The balloon dilation time (8-12 seconds of balloon dilation) was recorded. After the collection, the data were measured with LABSYSTEM analyzer by two senior doctors.

The measured indicators were as follows. (1) PR interval (the time interval from the start of the $\mathrm{P}$ wave to the start of the QRS wave); (2) RR interval (the time interval between the two QRS starting points); (3) Tpeak-Tend (TpTe) interval (the time interval from the peak of the $\mathrm{T}$ wave to the end of the T wave); (4) QRS time limit (the time interval from the beginning of the QRS wave to the end of the QRS); (5) QT interval (the time interval from the start of QRS wave to the end of T wave); (6) QTC interval: according to the QT interval, the equation was $\mathrm{QTC}=(\mathrm{QT} / \sqrt{\mathrm{RR}})$; (7) $\mathrm{R}$ wave amplitude (the distance from the upper edge of the QRS wave starting point to the top of the wave measured vertically); (8) $S$ wave amplitude (the distance from the bottom edge of the QRS wave starting point to the bottom of the wave measured vertically); (9) T wave amplitude (the distance from the starting point of QRS to the bottom of T wave is measured vertically).

2.4. Algorithm Performance Evaluation Experiment. Parts of the normal data and abnormal data in the single lead of China Cardiovascular Clinical Database (CDR) are selected as experimental data. Meanwhile, the GoogLeNet algorithm
[10] and the SqueezeNet algorithm [11] designed by the predecessors are introduced for comparison. With coronary angiography as the gold standard, the classification running time is recorded, and the algorithm performance evaluation indicators include the following ones.

(I) Parameter count (PC) is the corresponding learning parameters in the neural network convolutional layer. The smaller the parameter, the less the computer memory consumed.

(II) Accuracy (ACC) is a relatively comprehensive performance indicator that can directly reflect the performance of the model, which is expressed as follows with the confusion equation:

$$
\mathrm{ACC}=\frac{\mathrm{TP}+\mathrm{TN}}{\mathrm{TP}+\mathrm{TN}+\mathrm{FN}+\mathrm{FP}} .
$$

(III) Sensitivity (Se): the proportion of samples that are actually negative, and the equation is as follows:

$$
\mathrm{Se}=\frac{\mathrm{TP}}{\mathrm{TP}+\mathrm{FN}}
$$

(IV) Specificity (Sp) is the proportion of the actual positive sample data that is correctly predicted, and the equation is as follows:

$$
\mathrm{Sp}=\frac{\mathrm{TN}}{\mathrm{FP}+\mathrm{TN}}
$$

In (14)-(16), TP represents true positive, $\mathrm{FP}$ is false positive, $\mathrm{TN}$ represents true negative, and $\mathrm{FN}$ represents false negative. 
2.5. Statistical Methods. Data processing was analyzed by SPSS19.0 version statistical software, measurement data were represented by mean plus or minus standard deviation $(\bar{x} \pm s)$, and count data were represented by percentage (\%). The accuracy, sensitivity, specificity, running time, and number of parameters of LetNet-SoM algorithm, GoogLeNet algorithm, and SqueezeNet algorithm adopt paired $t$-test. The QRS, QTC, PR, RR, TpTe, QT, R wave, T wave, and $S$ wave indicators were compared by analysis of variance after anesthesia treatment. The difference was statistically significant when $P<0.05$.

\section{Results}

3.1. Contrast of Performance Indicators of Different Algorithms. Figure 3 reveals that the accuracy, sensitivity, and specificity of the LetNet-SoM algorithm were obviously higher in contrast to GoogLeNet algorithm and the SqueezeNet algorithm, with notable difference $(P<0.05)$. The running time and number of parameters of the LetNetSoM algorithm were obviously less in contrast to GoogLeNet algorithm and the SqueezeNet algorithm, with notable difference $(P<0.05)$.

3.2. Descriptive Statistics of Subjects'Basic Data. Figures 4 and 5 show that there were remarkably more male patients than females; the proportion of patients over 50 years old was the largest, followed by patients aged $40-50$ years. The proportion of patients who were hospitalized for longer than 10 months was the highest, followed by patients who were 5-10 months old. The proportion of patients with three-vessel disease was the highest, followed by patients with double-vessel disease. The proportion of patients whose intervened vessel was the anterior descending artery was the highest, followed by the patients with the right coronary artery intervened.

3.3. Indicator Comparison before and after Balloon Dilation. Figure 6 shows that QRS and QTC of patients after anesthesia treatment were obviously higher in contrast to that before treatment, with notable difference $(P<0.05)$. PR interval, RR interval, TpTe interval, and QT interval after anesthesia were not obviously different from those before treatment $(P>0.05)$. Figure 7 shows the changes of ECG before and after balloon dilatation, and the patient started balloon dilatation to block blood flow at $1 \mathrm{~s}$. At $3 \mathrm{~s}$, a relatively obvious positive change in QRS was observed, the duration of expansion was $8 \mathrm{~s}$, and QRS began to return to normal at $9 \mathrm{~s}$.

\subsection{Contrast of Amplitude Indicators before and after Balloon} Dilation. Figure 8 shows that the indicators of $\mathrm{R}$ wave and $\mathrm{T}$ wave of the patients after anesthesia treatment were obviously higher than those before the treatment, with notable difference $(P<0.05)$. The difference between the indicator $S$ wave after the anesthesia treatment and before the treatment was not obvious $(P>0.05)$.
3.5. Positive Rate of Various Indicators. Figure 9 shows that, among the various indicators, the positive rate of $S$ wave amplitude was the highest (68.15\%), followed by QRS $(60.52 \%)$ and QTC (51.36\%). However, the positive rates of other indicators were low.

\section{Discussion}

Cardiovascular disease is one of the common high-mortality foci in people's lives. The ECG is the most direct response form of the heart, but the ECG data itself is more complicated and difficult to predict, and the theoretical knowledge involved is diversified. Therefore, citing mathematical algorithms for data processing and classification is a hot spot for current scholars $[12,13]$. The ECG signal classification algorithm LetNet-SoM was constructed based on the lightweight neural network, and GoogLeNet and SqueezeNet algorithms were introduced for comparison. It was found that the accuracy, sensitivity, and specificity of the LetNet-SoM algorithm were obviously higher than those of the GoogLeNet and SqueezeNet algorithms, and the running time and the number of parameters were less than those of the GoogLeNet and SqueezeNet algorithms $(P<0.05)$. It was similar to the research results of Hung et al. [14], suggesting that, compared with the traditional algorithm, the constructed LetNet-SoM algorithm could reduce the running time and required parameters while maintaining the highprecision classification effect. After this algorithm was applied in the ECG diagnosis of patients with myocardial ischemia, it was found that QRS and QTC after treatment were notably higher than those before treatment $(P<0.05)$, which was similar to study results of Head et al. [15]. The length of QRS interval was related to the delay of ECG signal transmission in myocardial ischemic area. The length of the QTc interval was related to the distribution of ions inside and outside the cell and the decrease in the $\mathrm{pH}$ of the extracellular fluid. The results indicated that QRS and QTC were more sensitive to early myocardial ischemia.

The indicators of $\mathrm{R}$ wave and $\mathrm{T}$ wave of the patients after anesthesia treatment were notably higher in contrast to those before treatment, with notable difference $(P<0.05)$, and the indicator $S$ wave was not obviously different from those before treatment $(P>0.05)$. It was different from the findings of Burton et al. [16]. In patients with myocardial ischemia, the main systolic function was the increase in ventricular end-systolic volume, which triggered the increase in $\mathrm{R}$ wave, but the reason for the increase in $\mathrm{T}$ wave was not clear. The results indicated that $R$ wave and $T$ wave were more sensitive to early myocardial ischemia in patients, with obvious changes [17]. Among the various indicators, the positive rate of $\mathrm{S}$ wave amplitude was the highest (68.15\%), followed by QRS (60.52\%) and QTC (51.36\%). The positive rates of other indicators were low. It indicates that the diagnostic value of S wave, QRS, and QTC for myocardial ischemia was higher than other parameters. In summary, which was consistent with the research results of Collet et al. [18], it could be inferred that the ECG signal parameter S wave amplitude, QRS time limit, and QTC interval based on the LetNet-SoM algorithm had high clinical value for the diagnosis of early myocardial ischemia. 


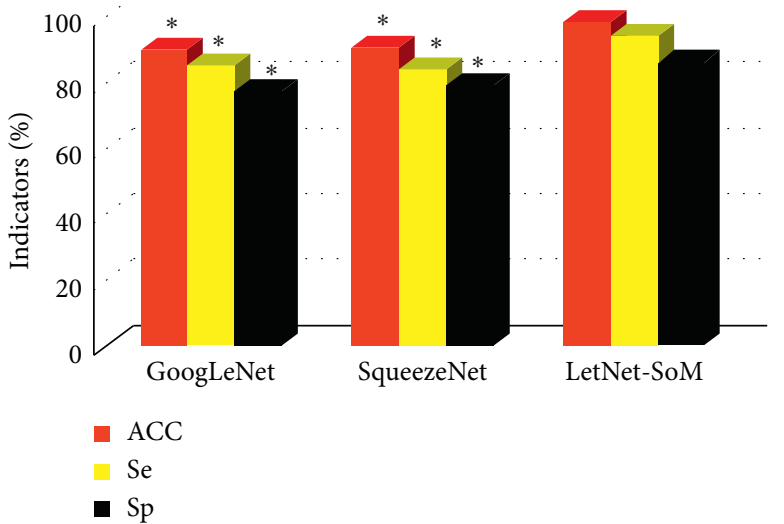

(a)

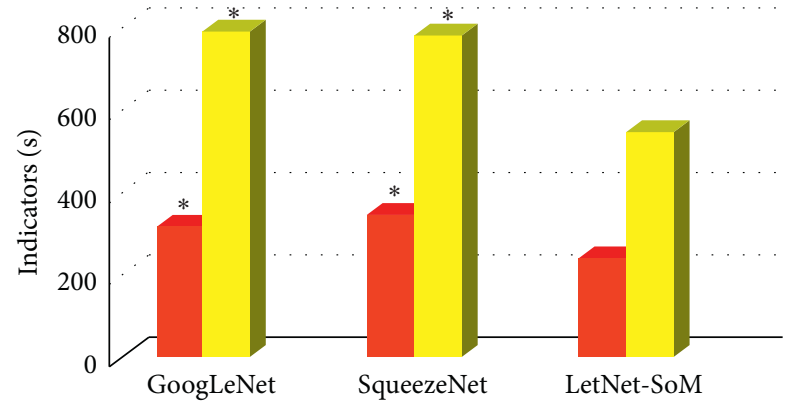

Time

PC

(b)

Figure 3: Contrast of performance indicators of different algorithms. (a) Accuracy, sensitivity, and specificity. (b) Running time and number of parameters. ${ }^{*}$ indicated notable difference when compared to LetNet-SoM $(P<0.05)$.

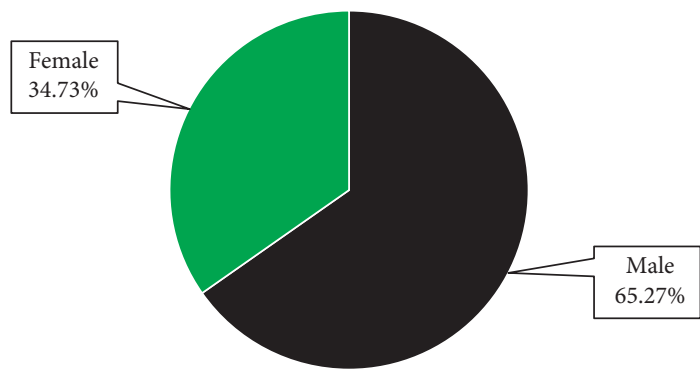

- Male

- Female

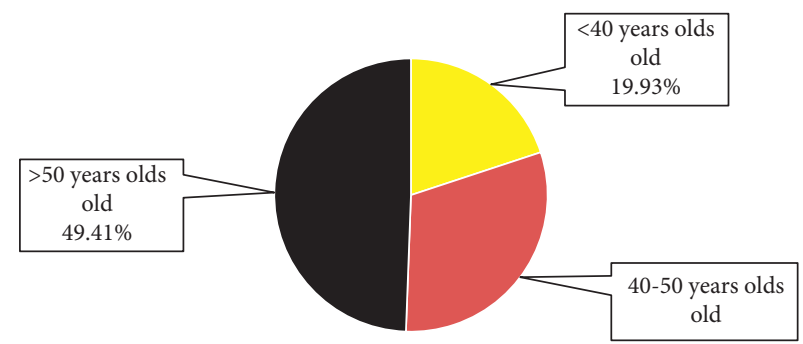

1. $<40$ years old

- 40-50 years old

- >50 years old

(a)

(b)

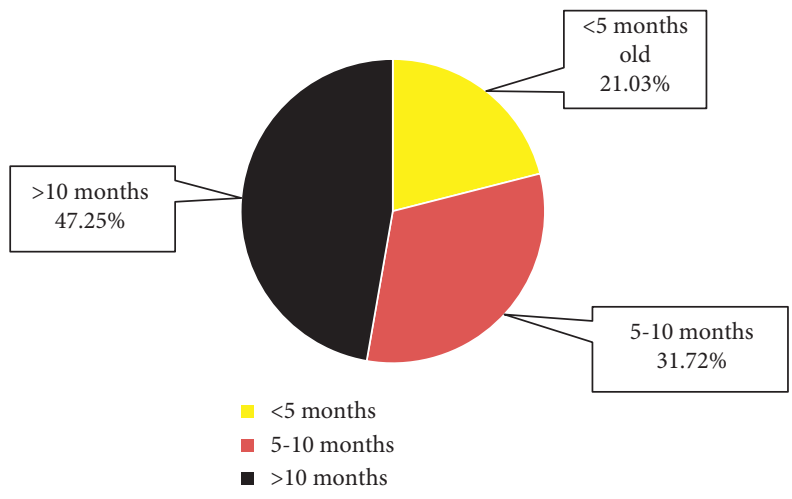

(c)

Figure 4: Descriptive statistics of subjects' gender, age, and duration of hospital stay. (a) The gender ratio of patients; (b) the age ratio of patients; (c) the duration of hospital stay. 


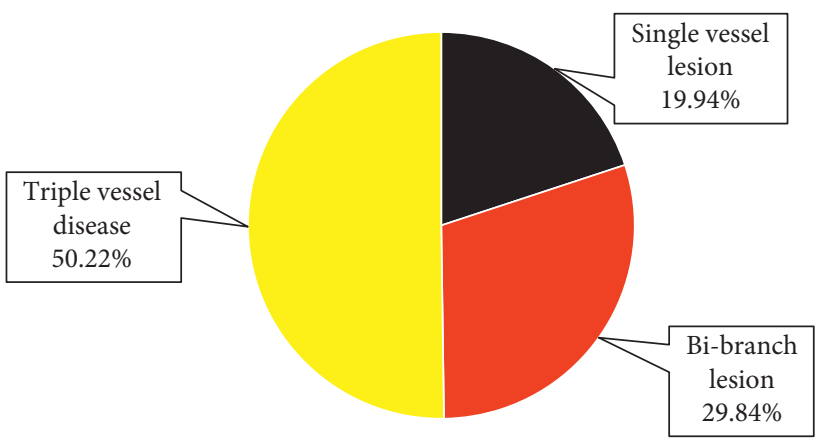

- Single vessel lesion

- Bi-branch lesion

- Triple vessel disease

(a)

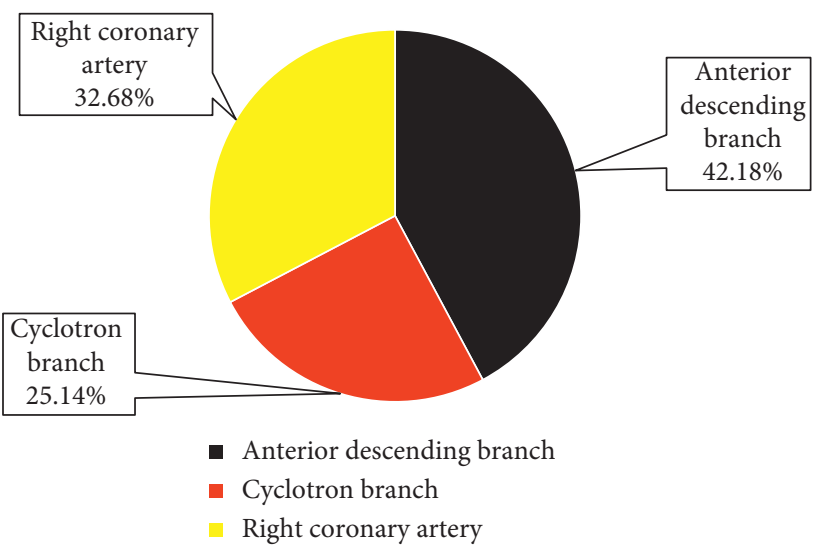

(b)

Figure 5: Descriptive statistics of subjects' vascular disease and intervention of vessels. (a) The patient's vascular disease; (b) the patient's intervention of vessels.

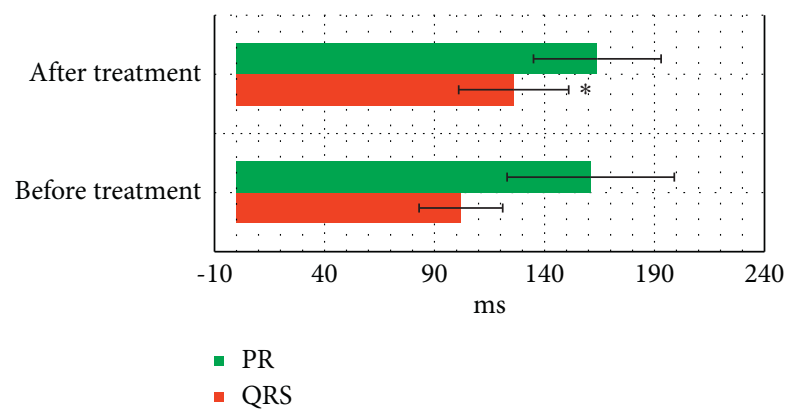

(a)

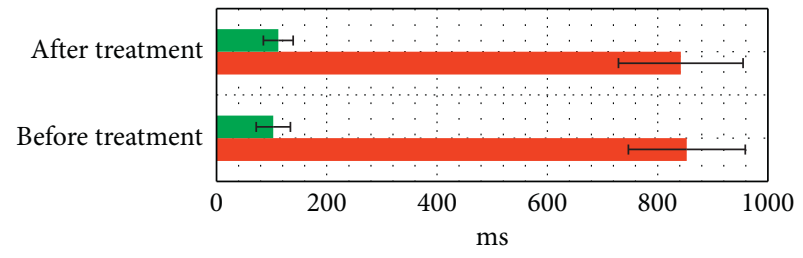

- $\mathrm{TpTe}$

- $\mathrm{RR}$

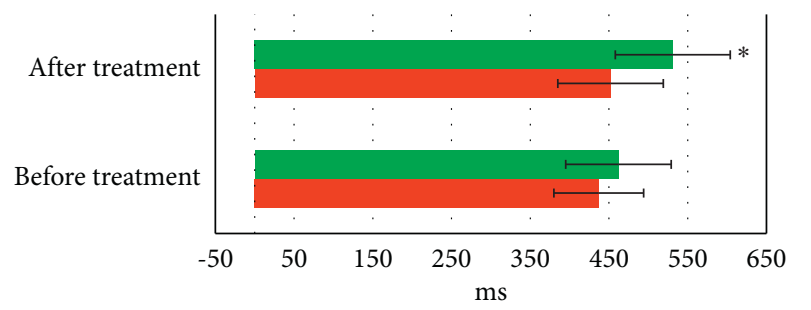

- QTC

- QT

(c)

FIGURE 6: Comparison of interval indicators before and after balloon dilation. (a) The patient's QRS and PR interval; (b) the patient's RR interval and TpTe interval; (c) the patient's QT interval and QTC. *indicates notable difference when compared to that before treatment $(P<0.05)$. 


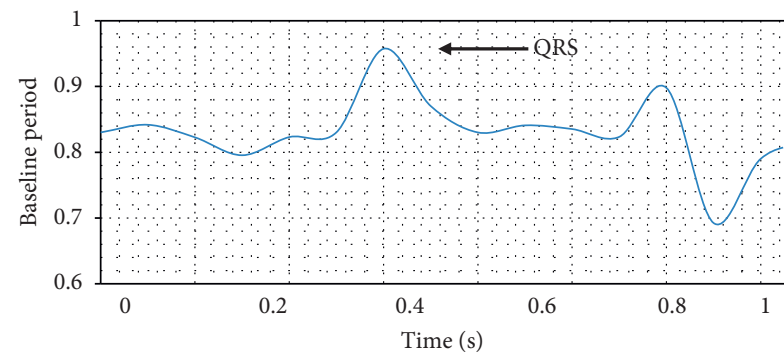

(a)

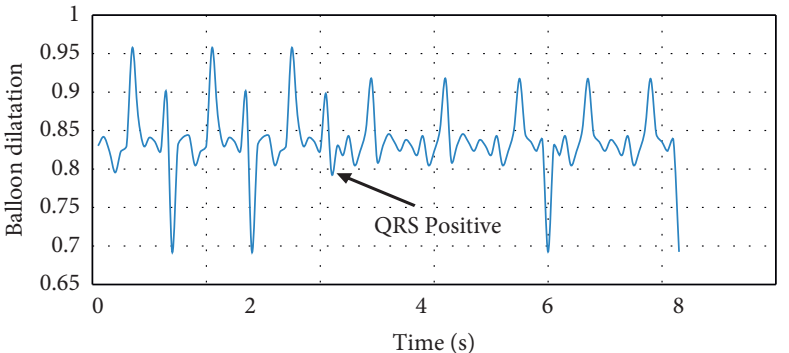

(b)

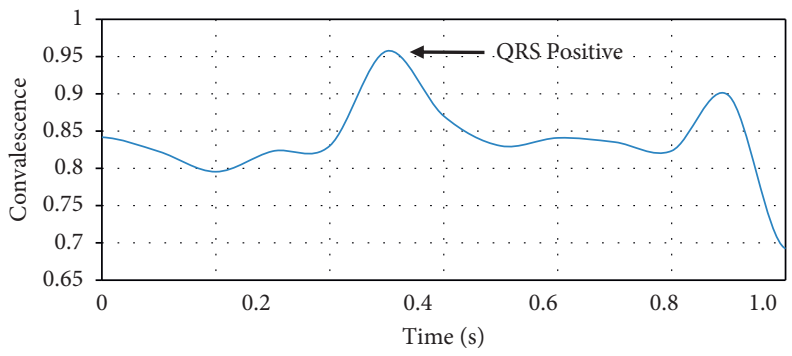

(c)

FIGURE 7: ECG changes before and after balloon dilation. (a) The ECG change at $1 \mathrm{~s}$ during the baseline period; (b) the ECG change during the balloon dilation period; (c) the ECG change during the recovery period.

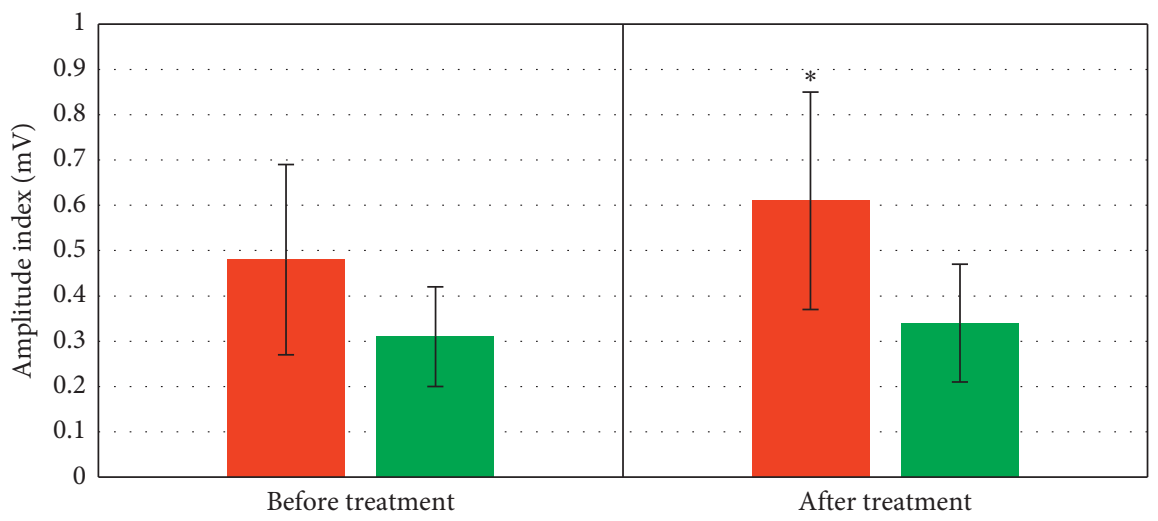

$\mathrm{R}$

- S

(a)

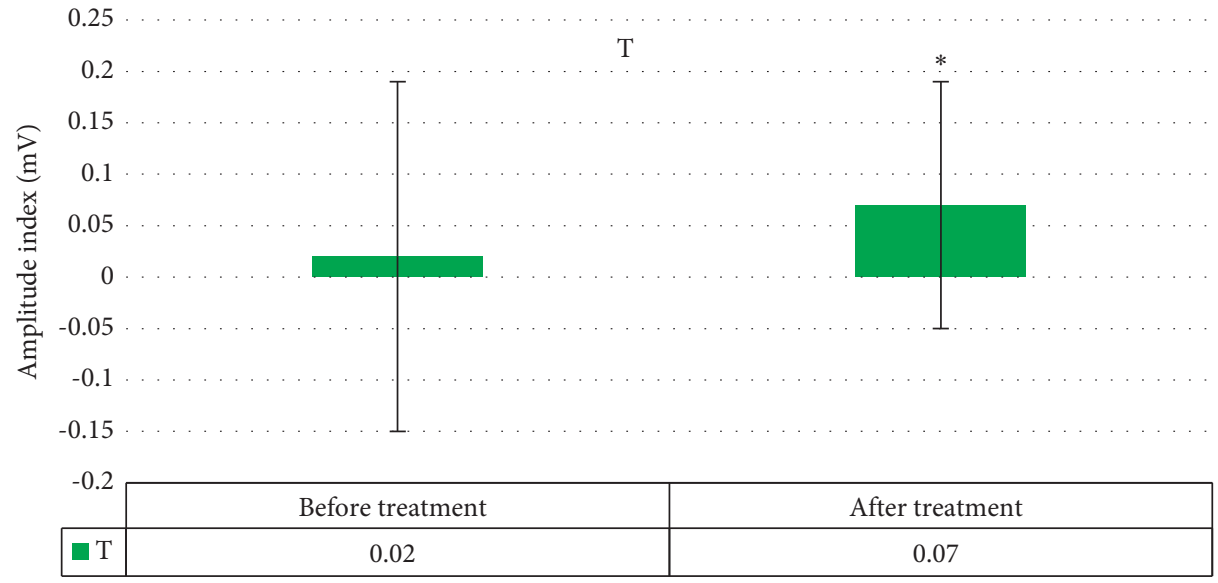

(b)

FIGURE 8: Comparison of amplitude indicators before and after balloon dilation. (a) Indicators of the patient's R wave amplitude and S wave amplitude; (b) indicators of the patient's T wave amplitude. ${ }^{*}$ indicates notable difference when compared to that before treatment $(P<0.05)$. 


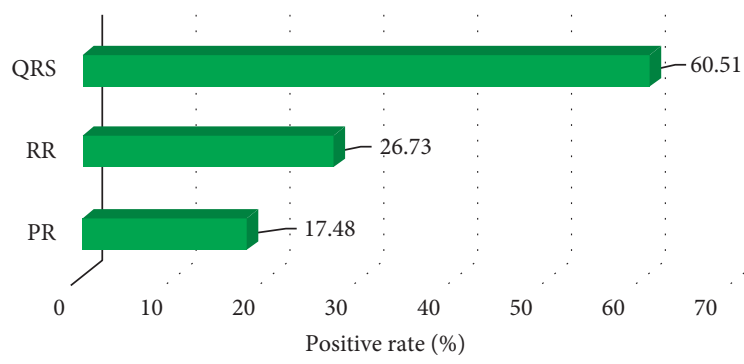

(a)

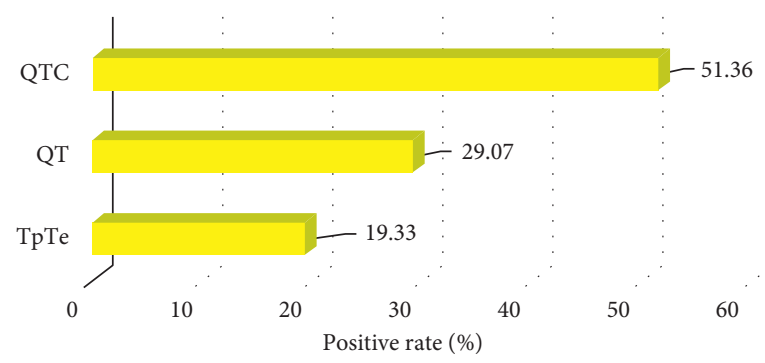

(b)

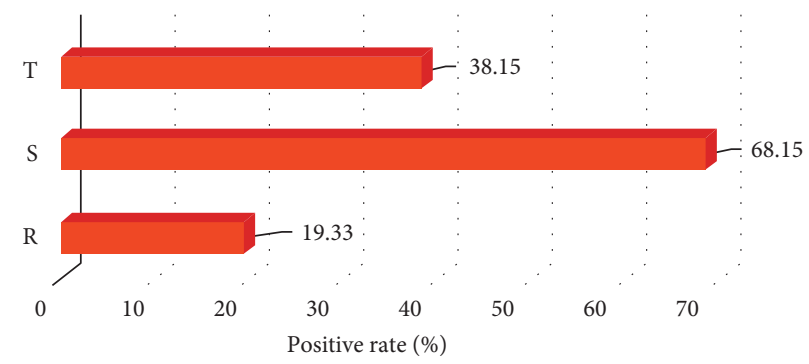

(c)

FIgURE 9: The positive rate of various indicators. (a) QRS, PR interval, and RR interval; (b) TpTe interval, QT interval, and QTC; (c) R wave, $\mathrm{S}$ wave, and $\mathrm{T}$ wave.

\section{Conclusion}

Based on the lightweight neural network, the ECG signal classification algorithm LetNet-SoM was constructed, and GoogLeNet and SqueezeNet algorithms were introduced for simulation and comparison, and they were adopted in the ECG diagnosis of patients with myocardial ischemia. The results revealed that, compared with the traditional algorithm, the LetNet-SoM algorithm could reduce the running time and the required parameters while maintaining the high-precision classification effect. The ECG signal parameter S wave amplitude, QRS time limit, and QTC interval based on LetNet-SoM algorithm had high clinical value for the diagnosis of early myocardial ischemia. However, the time of studied myocardial ischemia is relatively short, which is different from that of clinical patients. Subsequently, more extensive ECG signal samples are considered to be collected, to further analyze the positive status of each indicator. In conclusion, this study provides a theoretical basis for the classification of cardiac map data in the diagnosis of myocardial ischemic patients.

\section{Data Availability}

The data used to support the findings of this study are available from the corresponding author upon request.

\section{Conflicts of Interest}

The authors declare no conflicts of interest.

\section{References}

[1] S. Ansari, N. Farzaneh, M. Duda et al., "A review of automated methods for detection of myocardial ischemia and infarction using electrocardiogram and electronic health records," $R e$ views in Biomedical Engineering, vol. 10, pp. 264-298, 2017.
[2] A. Bayés de Luna, D. Rovai, G. Pons Llado et al., "The end of an electrocardiographic dogma: a prominent $\mathrm{R}$ wave in $\mathrm{V} 1$ is caused by a lateral not posterior myocardial infarction-new evidence based on contrast-enhanced cardiac magnetic resonance-electrocardiogram correlations," European Heart Journal, vol. 36, no. 16, pp. 959-964, 2015.

[3] H. Yang, A. Fayad, A. Chaput, S. Oake, A. D. Chan, and M. L. Crossan, "Postoperative real-time electrocardiography monitoring detects myocardial ischemia: a case report. Le monitorage électrocardiographique postopératoire en temps réel pour détecter l'ischémie myocardique: une présentation de cas," Canadian Journal of Anaesthesia, vol. 64, no. 4, pp. 411-415, 2017.

[4] R. B. Esper, M. E. Farkouh, E. E. Ribeiro et al., "Syntax score in patients with diabetes undergoing coronary revascularization in the freedom trial," Journal of the American College of Cardiology, vol. 72, no. 23 Pt A, pp. 2826-2837, 2018.

[5] Y. Fakhri, M. Sejersten, M. M. Schoos et al., "Electrocardiographic scores of severity and acuteness of myocardial ischemia predict myocardial salvage in patients with anterior ST-segment elevation myocardial infarction," Journal of Electrocardiology, vol. 51, no. 2, pp. 195-202, 2018.

[6] P. Valensi and C. Meune, "Congestive heart failure caused by silent ischemia and silent myocardial infarction: diagnostic challenge in type 2 diabetes. Kongestive herzinsuffizienz durch stumme ischämie und stummen herzinfarkt: diagnostische herausforderung bei typ-2-diabetes," Herz, vol. 44, no. 3, pp. 210-217, 2019.

[7] B. H. West, C. G. Low, B. B. Bista et al., "Significance of coronary artery calcium found on non-electrocardiogramgated computed tomography during preoperative evaluation for liver transplant," The American Journal of Cardiology, vol. 124, no. 2, pp. 278-284, 2019.

[8] D. J. F. M. Thuijs, A. P. Kappetein, P. W. Serruys et al., "Percutaneous coronary intervention versus coronary artery bypass grafting in patients with three-vessel or left main coronary artery disease: 10-year follow-up of the multicentre 
randomised controlled SYNTAX trial," Lancet, vol. 394, no. 10206, pp. 1325-1334, 2019.

[9] Y. X. Yin, W. Gao, X. Y. Li et al., "Insertion of peripherally inserted central catheters with intracavitary electrocardiogram guidance: a randomized multicenter study in China," The Journal of Vascular Access, vol. 20, no. 5, pp. 524-529, 2019.

[10] M. Ronzhina, V. Olejnickova, T. Stracina et al., "Effect of increased left ventricle mass on ischemia assessment in electrocardiographic signals: rabbit isolated heart study," BMC Cardiovascular Disorders, vol. 17, no. 1, p. 216, 2017.

[11] A. Shvilkin, E. R. Ellis, E. V. Gervino, A. D. Litvak, A. E. Buxton, and M. E. Josephson, "Painful left bundle branch block syndrome: clinical and electrocardiographic features and further directions for evaluation and treatment," Heart Rhythm, vol. 13, no. 1, pp. 226-232, 2016.

[12] E. S. Yu, J. J. Lange, A. Broor, and K. Kutty, “Acute pancreatitis masquerading as inferior wall myocardial infarction: a review," Case Reports in Gastroenterology, vol. 13, no. 2, pp. 321-335, 2019.

[13] G. N. Levine, E. R. Bates, J. C. Blankenship et al., "2015 ACC/ AHA/SCAI focused update on primary percutaneous coronary intervention for patients with ST-elevation myocardial infarction: an update of the 2011 ACCF/AHA/SCAI guideline for percutaneous coronary intervention and the $2013 \mathrm{ACCF} /$ AHA guideline for the management of ST-elevation myocardial infarction," Journal of the American College of Cardiology, vol. 67, no. 10, pp. 1235-1250, 2016.

[14] M. Y. Hung, N. G. Kounis, M. Y. Lu, and P. Hu, "Myocardial ischemic syndromes, heart failure syndromes, electrocardiographic abnormalities, arrhythmic syndromes and angiographic diagnosis of coronary artery spasm: literature review," International Journal of Medical Sciences, vol. 17, no. 8, pp. 1071-1082, 2020.

[15] S. J. Head, M. Milojevic, J. Daemen et al., "Mortality after coronary artery bypass grafting versus percutaneous coronary intervention with stenting for coronary artery disease: a pooled analysis of individual patient data," Lancet, vol. 391, no. 10124, pp. 939-948, 2018.

[16] B. M. Burton, K. K. Aras, W. W. Good, J. D. Tate, B. Zenger, and R. S. MacLeod, "A framework for image-based modeling of acute myocardial ischemia using intramurally recorded extracellular potentials," Annals of Biomedical Engineering, vol. 46, no. 9, pp. 1325-1336, 2018.

[17] D. F. Miranda, A. S. Lobo, B. Walsh, Y. Sandoval, and S. W. Smith, "New insights into the use of the 12-lead electrocardiogram for diagnosing acute myocardial infarction in the emergency department," Canadian Journal of Cardiology, vol. 34, no. 2, pp. 132-145, 2018.

[18] C. Collet, Y. Onuma, Y. Miyazaki, M. A. Morel, and P. W. Serruys, "Integration of non-invasive functional assessments with anatomical risk stratification in complex coronary artery disease: the non-invasive functional SYNTAX score," Cardiovascular Diagnosis and Therapy, vol. 7, no. 2, pp. 151-158, 2017. 